\title{
2+1D simulations of pre-equilibrium stage with QCD kinetic theory
}

\author{
Aleksi Kurkela \\ Theoretical Physics Department, CERN, Geneva, Switzerland \\ Faculty of Science and Technology, University of Stavanger, 4036 Stavanger, Norway \\ E-mail: a.k@cern.ch
}

\begin{abstract}
Aleksas Mazeliauskas*
Institut für Theoretische Physik, Universität Heidelberg, Heidelberg 69120, Germany

E-mail: a.mazeliauskas@thphys.uni-heidelberg.de
\end{abstract}

Jean-François Paquet

Department of Physics, Duke University, Durham, NC 27708, USA

E-mail: jeanfrancois.paquet@duke.edu

\section{Sören Schlichting}

Fakultät für Physik, Universität Bielefeld, D-33615 Bielefeld, Germany

E-mail: sschlichting@physik. uni-bielefeld.de

\section{Derek Teaney}

Department of Physics and Astronomy, Stony Brook University, Stony Brook, NY 11794, USA

E-mail: derek.teaneyestonybrook. edu

\begin{abstract}
The produced matter in the high energy nuclear collisions reinteracts and forms a plasma which ultimately equilibrates and exhibits collective hydrodynamic flow. The connection between the early gluon production in classical field simulations and hydrodynamic expansion at later times is given by the QCD kinetic theory. In this work we construct a set of non-equilibrium Green functions calculated in QCD kinetic theory to provide a practical tool to propagate energy and momentum perturbations from the early initial state to a time when hydrodynamics becomes applicable. We demonstrate with a realistic simulation of a heavy ion collisions that such linearized kinetic propagator (called $\mathrm{K} \varnothing \mathrm{MP} \varnothing \mathrm{ST}$ ) provides the smooth transition from the classical fields to hydrodynamics. KøMPøST can be easily incorporated into existing hydrodynamic simulations improving the early time description of heavy ion collisions.
\end{abstract}

International Conference on Hard and Electromagnetic Probes of High-Energy Nuclear Collisions 30 September - 5 October 2018

Aix-Les-Bains, Savoie, France

${ }^{*}$ Speaker. 


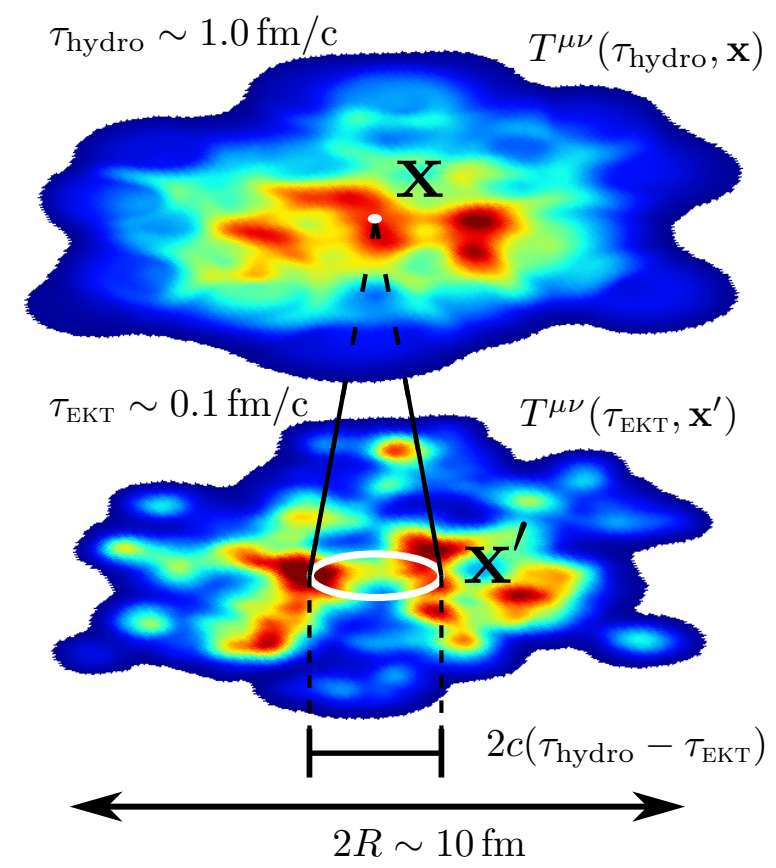

Figure 1: Transverse energy density profile in a nucleus-nucleus collisions before and after kinetic preequilibrium evolution. White circle indicates the size of local causal circle affecting the point $x$ in the future. Figure adapted from [12]

\section{Introduction}

One of the most striking results of heavy ion physics research in the last couple decades has been the observation that the quark-gluon plasma (QGP) behaves as viscous relativistic fluid with very small viscosity over entropy ratio $\eta / s$ [1]. How such macroscopic medium description can arise from the microscopic QCD degrees of freedom is still an actively researched topic [2,3]. In the weak coupling limit, the path to equilibrium has been proposed in the seminal "bottom-up" thermalization paper [4]. Taking over the semi-classical description of initial gluon fields in the color glass condensate (CGC) framework [5, 6, 7, 8], the weakly coupled QCD kinetic theory [9] uses particle scatterings to describe the approach to thermal and hydrodynamic equilibrium [10]. In this work we employ the weakly coupled QCD kinetic theory to describe the pre-equilibrium evolution of non-trivial transverse perturbations in event-by-event nucleus-nucleus collisions [11, 12] and present a practical framework (called KøMPøST) to perform this evolution [13].

\section{Non-equilibrium response from kinetic theory}

In a weak-coupling equilibration picture, the characteristic timescale for the transmutation of degrees of freedom from color-fields to particles is $\tau_{\mathrm{EKT}} \sim 1 / Q_{s} \sim 0.1 \mathrm{fm}$, where $Q_{s} \sim 2 \mathrm{GeV}$ is the gluon saturation scale, while the transition from kinetic to hydrodynamic description occurs at $\tau_{\text {hydro }} \sim 1 \mathrm{fm} \mathrm{[14].} \mathrm{The} \mathrm{short} \mathrm{duration} \mathrm{of} \mathrm{kinetic} \mathrm{pre-equilibrium} \mathrm{stage,} \mathrm{by} \mathrm{causality,} \mathrm{restricts} \mathrm{the}$ equilibration in a local causal patch which is much smaller than the transverse nucleus size

$$
R \gg c\left|\tau_{\text {hydro }}-\tau_{\mathrm{EKT}}\right| .
$$




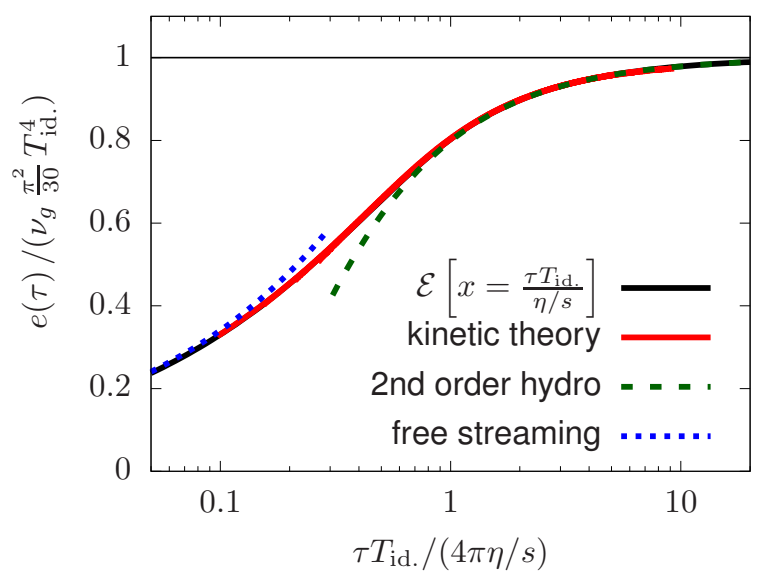

Figure 2: The universal scaling function, Eq. (2.3), fit to the kinetic theory evolution. The early time behavior is approximately described by free streaming, while the late time behavior matches smoothly onto the second order hydrodynamic asymptotics. Figure adopted from [11].

Within the causal patch shown in Fig. 1, the energy-momentum tensor can be decomposed in a homogeneous background part and (small) transverse perturbations around it

$$
T^{\mu v}\left(\tau_{\mathrm{EKT}}, \mathbf{x}^{\prime}\right)=\bar{T}_{\mathbf{x}}^{\mu v}\left(\tau_{\mathrm{EKT}}\right)+\delta T_{\mathbf{x}}^{\mu v}\left(\tau_{\mathrm{EKT}}, \mathbf{x}^{\prime}\right) .
$$

The equilibration of homogeneous, boost-invariant energy-momentum tensor $\bar{T}^{\mu v}=\left(e, P_{T}, P_{T}, P_{L} / \tau^{2}\right)$ has been studied in pure-glue kinetic theory previously [10]. The initially anisotropic pressures $P_{T} \gg P_{L}$ isotropize due to particle scatterings and start to obey the hydrodynamic constitutive equations, while the energy density approaches the ideal hydrodynamic behavior $e=v_{g} \frac{\pi^{2}}{30} T_{\mathrm{id}}^{4} \sim$ $\tau^{-4 / 3}$ [11]. Deviations of the energy density evolution in kinetic theory from the ideal expectation is shown in Fig. 2, and it can be parametrized by a scaling function $\mathscr{E}(x)$

$$
e(\tau)=v_{g} \frac{\pi^{2}}{30} T_{\text {id. }}^{4} \mathscr{E}\left[x=\frac{\tau T_{\text {id. }}}{\eta / s}\right] .
$$

The scaling variable $x=\frac{\tau T_{\text {id }}}{\eta / s}$ measures time in units of kinetic relaxation time $\tau_{R}=\eta / s T_{\text {id. }}$ and the scaling function $\mathscr{E}(x)$ remains remarkably unchanged for a substantial range of parameters (e.g. the value of the coupling constant or initial energy density) [11]. The transverse and longitudinal pressures are then self-consistently determined from the equation of motion for Bjorken expansion, i.e. $\partial_{\tau}(\tau e)=-P_{L}$, and the tracelessness of the conformal energy-momentum tensor $-e+2 P_{T}+$ $P_{L}=0$.

The pre-equilibrium evolution of transverse perturbations $\delta T^{\mu v}$ can be described by solving the linearized Boltzmann equation coupled to the homogeneous background [15, 11]. From the numerical kinetic theory simulations we constructed the non-equilibrium response functions $G_{\alpha \beta}^{\mu v}\left(\mathbf{x}, \mathbf{x}_{0}, \tau, \tau_{0}\right)$ which propagate initial fluctuations associated with energy $\left(\delta T^{\tau \tau}\right)$ and momentum $\left(\delta T^{\tau i}\right)$ perturbations

$$
\frac{\delta T^{\mu \nu}(\tau, \mathbf{x})}{\bar{T}_{\mathbf{x}}^{\tau \tau}(\tau)}=\frac{1}{\bar{T}_{\mathbf{x}}^{\tau \tau}\left(\tau_{0}\right)} \int d^{2} \mathbf{x}_{0} G_{\alpha \beta}^{\mu \nu}\left(\mathbf{x}, \mathbf{x}_{0}, \tau, \tau_{0}\right) \delta T_{\mathbf{x}}^{\alpha \beta}\left(\tau_{0}, \mathbf{x}_{0}\right)
$$




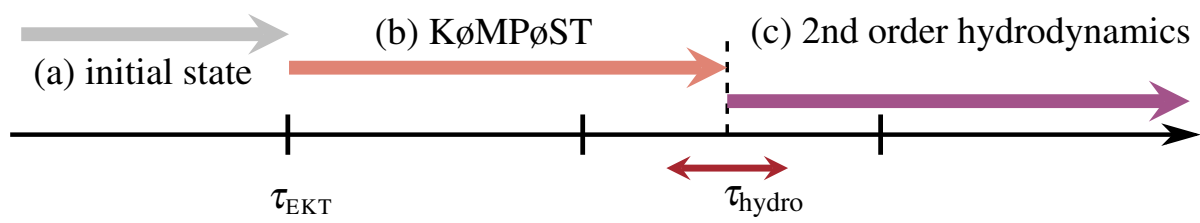

Figure 3: Workflow diagram of multi-stage heavy ion simulation with pre-equilibrium evolution. (a) Initial state provided by MC-Glauber prametrization or dynamical CGC evolution. (b) The equilibration of initial energy-momentum tensor with linearized kinetic theory propagator KøMPøST. (c) The viscous hydrodynamic simulation of QGP expansion.

Similarly to the background evolution, the response functions do not depend on the coupling constant or background energy density independently, but only through the dimensionless scaling variable $\frac{\tau T_{\mathrm{idd}}}{\eta / s}$. Therefore tabulated values of response functions can be used in event-by-event preequilibrium propagation of initial conditions.

\section{Event-by-event pre-equilibrium propagator-KøMPøST}

The kinetic equilibration scaling curve Eq. (2.3) and the non-linear response function, Eq. (2.4), encompasses the essential information about the out-of-equilibrium evolution of the energy-momentum tensor $T^{\mu v}$. Using them we constructed a linearized kinetic pre-equilibrium propagator-called $\mathrm{K} \varnothing \mathrm{MP} \varnothing \mathrm{ST}$ - which is publicly available and can be straightforwardly incorporated in modern eventby-event simulations of heavy ion collisions (see Fig. 3) [13].

The initialization of KøMPøST is done by providing $T^{\mu v}\left(\tau_{\mathrm{EKT}}, \mathbf{x}\right)$ at some early time $\tau_{\mathrm{EKT}} \sim$ $0.1 \mathrm{fm}$. In the "bottom-up" thermalization the initial state is described by the CGC effective theory and therefore KøMPøST can be naturally paired with the initial energy-momentum tensor generated by the dynamical IP-Glasma model [16, 17]. However, one can also initialize KøMPøST with initial state parametrization models, e.g. $\mathrm{T}_{\mathrm{R}}$ ENTo [18], which provide event-by-event fluctuation of initial energy density. In this case, the energy-momentum tensor at early times can be parametrized by $T^{\mu v}(\tau, \mathbf{x})=\operatorname{diag}(e(\tau, \mathbf{x}), e(\tau, \mathbf{x}) / 2, e(\tau, \mathbf{x}) / 2,0)$.

The pre-equilibrium evolution in KøMPøST is done separately for the local background and perturbations, Eq. (2.2). In practice the local background energy density $\bar{T}^{\tau \tau}$ is found by Gaussian averaging with typical width $2 \sigma=\left|\tau_{\text {hydro }}-\tau_{\mathrm{EKT}}\right|$. Using the initial $\bar{T}^{\tau \tau}, \tau_{\mathrm{EKT}}$, and $\eta / s$ one then determines the final background energy density and pressures at $\tau_{\text {hydro }}$ from the scaling curve Eq. (2.3). The difference $\delta T_{\mathbf{x}}^{\mu v}\left(\tau, \mathbf{x}^{\prime}\right)=T^{\mu v}\left(\tau, \mathbf{x}^{\prime}\right)-\bar{T}_{x}^{\mu v}(\tau)$ is treated as linearized perturbation. KøMPøST propagates initial energy $\left(\delta T^{\tau \tau}\right)$ and transverse momentum $\left(\delta T^{\tau i}\right)$ components using the convolution with the response functions in Eq. (2.4), while the shear-stress perturbations $\delta T^{i j}$ — which do not correspond to conserved charges—are neglected. The final energy-momentum tensor $T^{\mu v}\left(\mathbf{x}, \tau_{\text {hydro }}\right)$ is constructed as a sum of the background $\bar{T}^{\mu v}\left(\mathbf{x}, \tau_{\text {hydro }}\right)$ and perturbations $\delta T^{\mu v}\left(\mathbf{x}, \tau_{\text {hydro }}\right)$, which can be passed to the subsequent hydrodynamic evolution. In certain cases, e.g. on the edge of the fireball where gradients are especially large, the linearized approach fails and the constructed energy-momentum tensor does not have a well-defined fluid-frame. In these limited instances the kinetic response is regulated to facilitate the hydrodynamic evolution, but this does not affect the physical observables. 

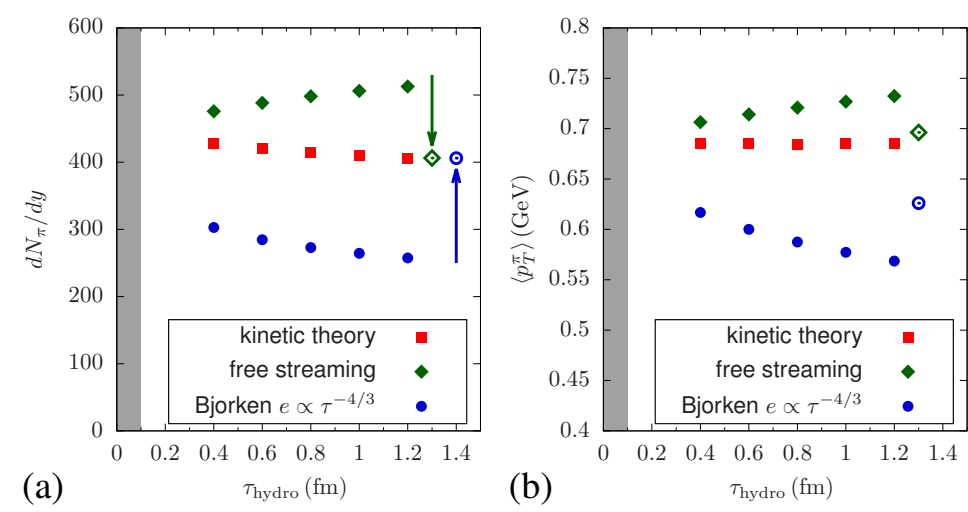

(b)

Figure 4: (a) Pion multiplicity (b) mean $p_{T}$ and (c) elliptic flow with varying duration of KøMPøST and free-streaming pre-equilibrium evolution. Figure adapted from [11]

\section{Results and discussion}

A theoretically consistent pre-equilibrium evolution should seamlessly match the later time hydrodynamics and the final observables should be insensitive to the variation of the cross-over time $\tau_{\text {hydro }}$ in the overlap region (see Fig. 3). In Fig. 4 we show the final pion multiplicity per unit rapidity $d N / d y$, mean $p_{T}$ and $v_{2}$ after the combined KøMPøST and hydrodynamic evolution with the variable cross-over time $\tau_{\text {hydro }}=0.4-1.2 \mathrm{fm}$. The initial conditions were generated using MC-Glauber parametrization and KøMPøST was initialized at $\tau_{\mathrm{EKT}}=0.1 \mathrm{fm}$. Since initially the transverse flow is zero only energy response functions were used. The red points in Fig. 4 correspond to hadronic observables after varying duration of kinetic pre-equilibrium, which are manifestly constant within few percents. We also compared KøMPøST results with two alternative pre-equilibrium scenarios: free-streaming and ideal rescaling. The green points show the results for collisionless free-streaming evolution, which does not bring the system into equilibrium, but generate transverse flow. Because of lack of longitudinal pressure, energy density in free-streaming falls slower than in hydrodynamics and the final multiplicity is increasing with later switching times. Blue points show the results of hydrodynamic evolution with the initial energy density rescaled according to ideal power low $e \propto \tau^{-4 / 3}$, the energy-momentum tensor set to be isotropic, and no transverse flow added. As a result, the mean $p_{T}$ falls substantially with later hydro initialization time. The changing multiplicity can compensated by manually readjusting the initial energy density (see empty points in Fig. 4). The differences for mean $p_{T}$ and elliptic flow $v_{2}$ then becomes somewhat smaller. However, no such readjustments are needed for KøMPøST pre-equilibrium to keep all three hadronic observables approximately constant.

$\mathrm{K} \varnothing \mathrm{MP} \varnothing \mathrm{ST}$ provides a physically motivated pre-equilibrium evolution based on the microscopic dynamics of QCD. Already good matching to hydrodynamics, can be systematically improved by included fermionic degrees of freedom $[19,20]$ and extending the response formalism to inhomogeneous backgrounds.

\section{Acknowledgments}

This work was supported in part by the German Research Foundation (DFG) Collaborative Research Centre (SFB) 1225 (ISOQUANT) (A.M.) 


\section{References}

[1] W. Busza, K. Rajagopal and W. van der Schee, Ann. Rev. Nucl. Part. Sci. 68, 339 (2018) doi:10.1146/annurev-nucl-101917-020852 [arXiv:1802.04801 [hep-ph]].

[2] P. Romatschke, Phys. Rev. Lett. 120, 012301 (2018) doi:10.1103/PhysRevLett.120.012301 [arXiv:1704.08699 [hep-th]].

[3] A. Kurkela, U. A. Wiedemann and B. Wu, arXiv:1805.04081 [hep-ph].

[4] R. Baier, A. H. Mueller, D. Schiff and D. T. Son, Phys. Lett. B 502, 51 (2001) doi:10.1016/S0370-2693(01)00191-5 [hep-ph/0009237].

[5] E. Iancu, A. Leonidov and L. McLerran, hep-ph/0202270.

[6] E. Iancu and R. Venugopalan, In *Hwa, R.C. (ed.) et al.: Quark gluon plasma* 249-3363 doi:10.1142/9789812795533_0005 [hep-ph/0303204].

[7] F. Gelis, T. Lappi and R. Venugopalan, Int. J. Mod. Phys. E 16, 2595 (2007) doi:10.1142/S0218301307008331 [arXiv:0708.0047 [hep-ph]].

[8] F. Gelis, E. Iancu, J. Jalilian-Marian and R. Venugopalan, Ann. Rev. Nucl. Part. Sci. 60, 463 (2010) doi:10.1146/annurev.nucl.010909.083629 [arXiv:1002.0333 [hep-ph]].

[9] P. B. Arnold, G. D. Moore and L. G. Yaffe, JHEP 0301, 030 (2003) doi:10.1088/1126-6708/2003/01/030 [hep-ph/0209353].

[10] A. Kurkela and Y. Zhu, Phys. Rev. Lett. 115, 182301 (2015) doi:10.1103/PhysRevLett.115.182301 [arXiv:1506.06647 [hep-ph]].

[11] A. Kurkela, A. Mazeliauskas, J. F. Paquet, S. Schlichting and D. Teaney, arXiv:1805.00961 [hep-ph].

[12] A. Kurkela, A. Mazeliauskas, J. F. Paquet, S. Schlichting and D. Teaney, arXiv:1805.01604 [hep-ph].

[13] A. Kurkela, A. Mazeliauskas, J. F. Paquet, S. Schlichting and D. Teaney, https://github.com/KMPST/KoMPoST (2018).

[14] A. Kurkela, Nucl. Phys. A 956, 136 (2016) doi:10.1016/j.nuclphysa.2016.01.069 [arXiv:1601.03283 [hep-ph]].

[15] L. Keegan, A. Kurkela, A. Mazeliauskas and D. Teaney, JHEP 1608, 171 (2016) doi:10.1007/JHEP08(2016)171 [arXiv:1605.04287 [hep-ph]].

[16] B. Schenke, P. Tribedy and R. Venugopalan, Phys. Rev. Lett. 108, 252301 (2012) doi:10.1103/PhysRevLett.108.252301 [arXiv:1202.6646 [nucl-th]].

[17] B. Schenke, P. Tribedy and R. Venugopalan, Phys. Rev. C 86, 034908 (2012) doi:10.1103/PhysRevC.86.034908 [arXiv:1206.6805 [hep-ph]].

[18] J. S. Moreland, J. E. Bernhard and S. A. Bass, Phys. Rev. C 92, 011901 (2015) doi:10.1103/PhysRevC.92.011901 [arXiv:1412.4708 [nucl-th]].

[19] A. Kurkela and A. Mazeliauskas, arXiv:1811.03068 [hep-ph].

[20] A. Kurkela and A. Mazeliauskas, arXiv:1811.03040 [hep-ph]. 